\title{
Acidentes na primeira infância: contribuições da Enfermagem na construção de orientações preventivas
}

\author{
Accidents in early childhood: nursing contributions in the construction of preventive orientations \\ Accidentes en la primera infancia: contribuciones de la enfermería en la construcción de \\ orientaciones preventivas
}

Recebido: 27/01/2021 | Revisado: 02/02/2021 | Aceito: 04/02/2021 | Publicado: 10/02/2021

\author{
Danielle de Fátima Magalhães \\ ORCID: https://orcid.org/0000-0002-6863-3010 \\ Universidade Positivo, Brasil \\ E-mail: dannymagalhaes.s2@gmail.com \\ Kwanna Fiuza de Toledo Nobre \\ ORCID: https://orcid.org/0000-0002-4147-5315 \\ Universidade Positivo, Brasil \\ E-mail: kwanna_toledo@ hotmail.com \\ Laís Carolini Theis \\ ORCID: https://orcid.org/0000-0001-6346-8661 \\ Universidade Positivo, Brasil \\ E-mail: laistheis@gmail.com \\ Luciane Favero Basegio \\ ORCID: https://orcid.org/0000-0002-4486-0032 \\ Complexo Hospital de Clínicas da Universidade Federal do Paraná, Brasil \\ E-mail: lucianefavero@yahoo.com.br
}

\begin{abstract}
Resumo
Identificar os principais acidentes que acometem as crianças na primeira infância e propor orientações preventivas aos pais ou responsáveis a fim de minimizar a incidência de acidentes e reduzir eventuais danos à saúde da criança. Tratase de um estudo transversal, descritivo com abordagem quantitativa, realizado com crianças de zero a seis anos atendidas em um hospital de referência em trauma em uma capital do sul do Brasil, no período de julho a agosto de 2020. Os resultados evidenciaram que o perfil de crianças acometidas por acidentes predomina-se do sexo masculino $(55,2 \%)$, com idade média de 3,4 anos e a queda é o principal incidente que leva as crianças aos serviços de emergência, correspondendo a 69,1\%. A partir dos resultados evidenciados e levantamento do perfil de acidentes mais prevalentes, foi elaborado um material educativo para orientação aos pais, familiares e cuidadores na prevenção de futuros acidentes. Conclui-se que as crianças estão expostas diariamente a inúmeros riscos, sendo imprescindível o papel do profissional enfermeiro nas ações de educação em saúde para prevenção de acidentes com o intuito de disseminar conhecimento e reduzir agravos.
\end{abstract}

Palavras-chave: Prevenção de acidentes; Cuidados de enfermagem; Criança.

\begin{abstract}
Identify the main accidents that affect children in early childhood and propose preventive guidance to parents or guardians in order to minimize the incidence of accidents and reduce any damage to the child's health. This is a crosssectional, descriptive study with a quantitative approach, carried out with children aged zero to six years attended at a trauma reference hospital in a capital of southern Brazil, from July to August 2020. The results showed that the profile of children affected by accidents is predominantly male (55.2\%), with an average age of 3.4 years and falling is the main incident that takes children to emergency services, corresponding to $69.1 \%$. Based on the results evidenced and the survey of the most prevalent accident profile, an educational material was prepared to guide parents, family members and caregivers in the prevention of future accidents. It is concluded that children are exposed daily to innumerable risks, being essential the role of the professional nurse in health education actions for accident prevention in order to disseminate knowledge and reduce injuries.
\end{abstract}

Keywords: Accident Prevention; Nursing Care; Child.

\section{Resumen}

Identificar los principales accidentes que afectan a los niños en la primera infancia y proponer orientaciones preventivas a los padres o tutores con el fin de minimizar la incidencia de accidentes y reducir los daños a la salud del niño. Se trata de un estudio descriptivo transversal con abordaje cuantitativo, realizado con niños de cero a seis años atendidos en un hospital de referencia de trauma en una capital del sur de Brasil, de julio a agosto de 2020.Los resultados mostraron que el perfil de los niños Los afectados por accidentes son predominantemente masculinos 
$(55,2 \%)$, con una edad media de 3,4 años y las caídas son el principal incidente que lleva a los niños a los servicios de emergencia, correspondientes al $69,1 \%$. Con base en los resultados evidenciados y la encuesta del perfil de accidentes más prevalentes, se elaboró un material educativo para orientar a los padres, familiares y cuidadores en la prevención de futuros accidentes. Se concluye que los niños y niñas están expuestos diariamente a innumerables riesgos, siendo fundamental el papel del profesional de enfermería en las acciones de educación sanitaria para la prevención de accidentes con el fin de difundir conocimientos y reducir las lesiones.

Palabras clave: Prevención de Accidentes; Atención de Enfermeria; Niño.

\section{Introdução}

Acidente segundo a Organização Mundial da Saúde é "um acontecimento fortuito, geralmente danoso ou ainda um acontecimento independente da vontade humana provocado por uma força exterior que atua rapidamente e que se manifesta por um dano corporal ou mental”. Um evento não intencional, desastroso e evitável passível de prevenção (Who, 2009).

São considerados acidentes por causas externas de maior prevalência aqueles que englobam quedas, queimaduras, envenenamento, afogamento, sufocação/obstrução de vias aéreas, intoxicação e acidentes de trânsito, os quais representam um grave problema de saúde pública. Os danos ocasionados correspondem a uma das principais causas de mortalidade ou invalidez na primeira infância, capazes de acarretar sofrimento às crianças envolvidas, aos responsáveis, familiares, cuidadores, além de elevar os custos ao sistema de saúde. Fatos estes que podem ser evitados, uma vez que a maioria dos acidentes é passível de prevenção (Brasil, 2017a; Brasil, 2018).

A maioria dos acidentes ocorre devido à conjuntura das crianças na primeira infância, o que as torna mais vulneráveis a acidentes, decorrente da sua imaturidade, idade, curiosidade, desenvolvimento, integridade física, ambiente que são expostas e do estilo de vida da família. Envolvem ambos os sexos, todas as raças, qualquer classe social e econômica, porém, com incidências diferentes em algumas dessas categorias, ocorrendo mais em uma do que em outras (Araújo et al., 2017; Silva et al., 2017a).

Quanto aos locais dos acidentes, o ambiente doméstico é o de maior incidência, visto que é onde as crianças passam a maior parte de sua infância e por ser um ambiente que envolve múltiplos fatores intrapessoais, interpessoais, culturais e institucionais. O domicílio é caracterizado como um dos principais locais de acidentes que levam a internação hospitalar, seguido dos acidentes ocorridos nas vias públicas e no trânsito (Silva et al., 2017b).

Segundo dados do Sistema de Informações sobre Mortalidade em 2017, 3.197 crianças de zero a nove anos de idade morreram vítimas de acidentes por causas externas. Destes, 140 corresponderam a quedas, 713 a acidentes de transporte/trânsito, 1.402 a sufocação, afogamento e obstrução de vias aéreas. O restante envolveu outros tipos de acidentes, como queimaduras, intoxicações ou envenenamentos, entre outros. Os acidentes correspondem a aproximadamente $70 \%$ de todas as mortes de crianças que envolvem causas externas no Brasil. No mesmo ano e seguindo a mesma faixa etária, foram registradas 71.264 internações hospitalares no Sistema Único de Saúde por acidentes (Brasil, 2017b, Brasil, 2017c).

Os dados apresentados revelam a necessidade de prevenir acidentes envolvendo crianças, porém, poucas ações diligentes e efetivas são realizadas nos ambientes de saúde. Apesar da existência de políticas públicas e estratégias na Atenção Básica direcionadas a prevenção e promoção de acidentes, observa-se que estas não vêm se manifestando de forma satisfatória e eficiente, pois poucas ações voltadas para a educação em saúde são adotadas, devido ao déficit da atuação do enfermeiro e também da equipe de saúde (Brito et al., 2017; Faria et al., 2018; Silva \& Fernandes, 2019).

A falta de orientações adequadas aos pais, familiares e cuidadores, acarreta uma população desinformada e despreparada (Faria et al., 2018). É necessário que a equipe multiprofissional seja promotora de informações e participe ativamente na prevenção de acidentes, assumindo este problema no mesmo nível de prioridade que os demais assuntos que envolvam um desenvolvimento saudável. Sendo necessário falar sobre prevenção de acidentes nas consultas de enfermagem desde a puericultura assim como nos demais retornos de acompanhamento da criança (Araújo et al., 2017; Filócomo et al., 
2017).

Sendo assim, a elaboração de ações preventivas contribui para que medidas sejam colocadas em prática e aplicadas de forma efetiva, a fim de minimizar acidentes e seus impactos, principalmente aqueles que envolvem crianças na primeira infância. Dessa forma, esse estudo tem por objetivos identificar os principais acidentes que acometem as crianças na primeira infância e propor orientações preventivas aos pais ou responsáveis a fim de minimizar a incidência de acidentes e reduzir eventuais danos à saúde da criança.

\section{Metodologia}

Trata-se de um estudo transversal, de natureza quantitativa. O método quantitativo coleta dados por meio do uso de medições de grandezas e obtém-se por meio da metrologia, números com suas respectivas unidades. Este método permite com que sejam estabelecidas bases de dados que podem ser analisados por meio de técnicas matemáticas (Pereira et al., 2018).

O estudo foi realizado em uma unidade de pronto atendimento de um Hospital Escola referência no atendimento de urgência e emergência que atende adultos e crianças em diversas especialidades, localizado em uma capital do Sul do Brasil.

A coleta de dados ocorreu em duas etapas, sendo a primeira por meio de dados secundários disponíveis nos prontuários e registros de atendimentos de crianças atendidas no local de estudo. Os critérios para inclusão do estudo foram: registros de crianças com idade entre zero e seis anos completos de idade; devido a algum tipo de acidente, durante o período de 01 de julho a 31 de agosto do ano de 2020. Para coleta de dados utilizou-se um instrumento estruturado adaptado pelos autores, baseado no instrumento Viva Inquérito 2017 (Brasil, 2019). A busca das referidas informações nos prontuários foi realizada de forma reservada, sem cópias e/ou fotos, sendo transcritas as informações necessárias para o instrumento de coleta de dados, preservando a identificação dos pacientes.

A segunda etapa da coleta de dados deu-se por meio de entrevistas, com aplicação de instrumento estruturado adaptado pelos autores, baseado no instrumento Viva Inquérito 2017 (Brasil, 2019), no período de 23 a 31 de agosto, nos turnos matutino e vespertino. As entrevistas foram realizadas com pais ou responsáveis por crianças vítimas de acidentes que preencheram os seguintes critérios de inclusão: pais ou responsáveis de crianças entre zero e seis anos, 11 meses e 29 dias de idade, vítimas de acidentes; que foram atendidas no local de estudo; com idade superior a 18 anos.

Como critérios de exclusão elencaram-se: vítimas de lesões intencionais, como agressões, violência sexual, negligência/abandono, entre outras formas de violência; e ainda, situações em que o acesso aos dados dos atendimentos das crianças no prontuário eletrônico ou registros do hospital não estiveram disponíveis.

As entrevistas foram realizadas por duas pesquisadoras deste estudo em um espaço reservado, distante do público, de modo a preservar o momento que os envolvidos enfrentavam. Aos participantes foi explicado os objetivos, riscos e benefícios da pesquisa, e posterior assinatura do Termo de Consentimento Livre e Esclarecido, os dados foram coletados.

O anonimato dos participantes foi respeitado, sendo substituído o nome das crianças no prontuário e registros pela letra "C" e dos pais ou responsáveis pela letra "P", ambos seguidos de um numeral ordinal referente à sequência estabelecida de coleta. Cada participante levou em torno de dez minutos para responder ao instrumento.

Salienta-se que o planejamento inicial do estudo era realizar a coleta de dados apenas por meio de entrevistas e aplicação do questionário, entretanto, devido à situação pandêmica acometida pelo Coronavírus, houve uma mobilização para que a população evitasse busca por serviços de saúde em casos de baixo risco, bem como houve restrição de acesso de pesquisadores aos serviços de saúde para atividades de pesquisa.

Os dados foram analisados de forma descritiva por meio de frequências simples e médias aritméticas. Deste modo identificaram-se os tipos de acidentes mais prevalentes no local e no período, de acordo com a faixa etária, o sexo da criança, local de ocorrência do acidente, gravidade clínica, horário do acidente, lesões envolvidas e seguimento estabelecido, 
apresentados por meio de tabelas e gráficos.

A partir das informações coletadas foi elaborado um material suplementar com orientações preventivas de acidentes, disponibilizado à equipe de Enfermagem do setor e do hospital para serem entregues aos pais, responsáveis ou cuidadores, a fim de prevenir novos acidentes com as crianças na primeira infância.

Este trabalho foi apreciado pelo Comitê de Ética e Pesquisa da Universidade Positivo sob o parecer n ${ }^{\circ} 4.016 .580$ em 28 de maio de 2020 e pelo Comitê de Ética e Pesquisa da instituição coparticipante sob o parecer nº 4.112 .553 em 26 de junho de 2020. Obedecendo às exigências do Conselho Nacional de Saúde no que diz à execução de pesquisas com seres humanos nomeados pela Resolução 466/12 (Brasil, 2012).

\section{Resultados}

O estudo abordou 346 prontuários ou registros de atendimento e foram entrevistados 23 pais ou responsáveis por crianças vítimas de acidentes, totalizando 369 participantes. Destes, a maior prevalência correspondeu a crianças do sexo masculino com 204 (55,2\%), faixa etária média de três anos e quatro meses de idade, variando entre um mês e seis anos e nove meses.

A Tabela 1 revela que os acidentes acontecem predominantemente no período noturno entre as dezoito horas e meia noite $(43,1 \%)$. Em relação ao local de ocorrência de acidentes, 91,5\% dos prontuários não possuíam esse registro, mas os que apresentavam, a maior frequência foi no próprio local de moradia das crianças, dado confirmado nas entrevistas realizadas. Com relação ao dia da semana observou-se uma distribuição homogênea entre os dados.

Tabela 1 - Distribuição por horário de ocorrência do acidente, Curitiba/PR, 2020.

\begin{tabular}{lcc}
\hline Horário do Acidente & N & \% \\
\hline Período da manhã $(06 \mathrm{~h}-12 \mathrm{~h})$ & 34 & 9,2 \\
\hline Período da tarde $(12 \mathrm{~h}-18 \mathrm{~h})$ & 138 & 37,4 \\
\hline Período da noite $(18 \mathrm{~h}-00 \mathrm{~h})$ & 159 & 43,1 \\
\hline Período da madrugada $(00 \mathrm{~h}-06 \mathrm{~h})$ & 28 & 7,6 \\
\hline Sem registro & 10 & 2,7 \\
\hline Total & $\mathbf{3 6 9}$ & $\mathbf{1 0 0}$ \\
\hline
\end{tabular}

Fonte: dados da pesquisa (2020).

Na Tabela 2, a distribuição por tipo de acidente, identifica que queda é o principal incidente que leva as crianças aos serviços de emergência $(69,1 \%)$, seguida de trauma direto $(6 \%)$, tração (puxar a criança pelo braço - 3,8\%) e ferimento por objeto perfurante ou cortante (3\%). 
Research, Society and Development, v. 10, n. 2, e21010212415, 2021

(CC BY 4.0) | ISSN 2525-3409 | DOI: http://dx.doi.org/10.33448/rsd-v10i2.12415

Tabela 2 - Distribuição quanto ao tipo de acidente, Curitiba/PR, 2020.

\begin{tabular}{|c|c|c|}
\hline Tipo de Acidente & $\mathbf{N}$ & $\%$ \\
\hline Queda & 255 & 69,1 \\
\hline Atropelamento & 6 & 1,6 \\
\hline Queimadura & 1 & 0,3 \\
\hline Sufocação/engasgo & 5 & 1,4 \\
\hline Acidente de trânsito & 7 & 1,9 \\
\hline Entorse/Luxação ou fratura & 5 & 1,4 \\
\hline Queda de objeto sobre a criança & 10 & 2,7 \\
\hline Ferimento por objeto perfuro ou cortante & 11 & 3,0 \\
\hline Acidentes com animais & 10 & 2,7 \\
\hline Corpo estranho & 10 & 2,7 \\
\hline Trauma direto & 22 & 6,0 \\
\hline Amputação traumática & 1 & 0,3 \\
\hline Esmagamento/preensão & 4 & 1,1 \\
\hline Tração (puxar a criança pelo braço) & 14 & 3,8 \\
\hline Sem registro & 1 & 0,3 \\
\hline Outro acidente não especificado & 7 & 1,9 \\
\hline Total & 369 & 100 \\
\hline
\end{tabular}

Fonte: Dados da pesquisa (2020).

Com relação à origem das quedas, predominaram as quedas de outro nível (63,2\%), sendo estas provenientes da cama, berço e sofá $(21,3 \%)$, as não especificadas (14,2\%), de outras procedências (rede, vaso sanitário, outras mobílias, andador, carrinho, bebê conforto, cadeira/cadeirão, muro, barranco, janela, colo da mãe e automóvel - 13,5\%), de escada e degrau 
(6,3\%), de brinquedos (balança, motinho/triciclo, escorregador, cama elástica - 4,3\%) e bicicleta $(3,6 \%)$.

Em relação à natureza da lesão decorrente dos acidentes elencados, a maior frequência está relacionada a fraturas $(23,3 \%)$, acompanhado das contusões $(22,5 \%)$ e traumatismo crânio encefálico (22\%), conforme exposto na Figura 1. Dentre as complicações mais frequentes, a parte do corpo mais afetada foi a região da cabeça e face $(37,1 \%)$, seguido dos membros superiores $(34,4 \%)$ e membros inferiores $(12,4 \%)$.

Figura 1. Distribuição dos acidentes quanto à natureza da lesão, Curitiba/PR, 2020.

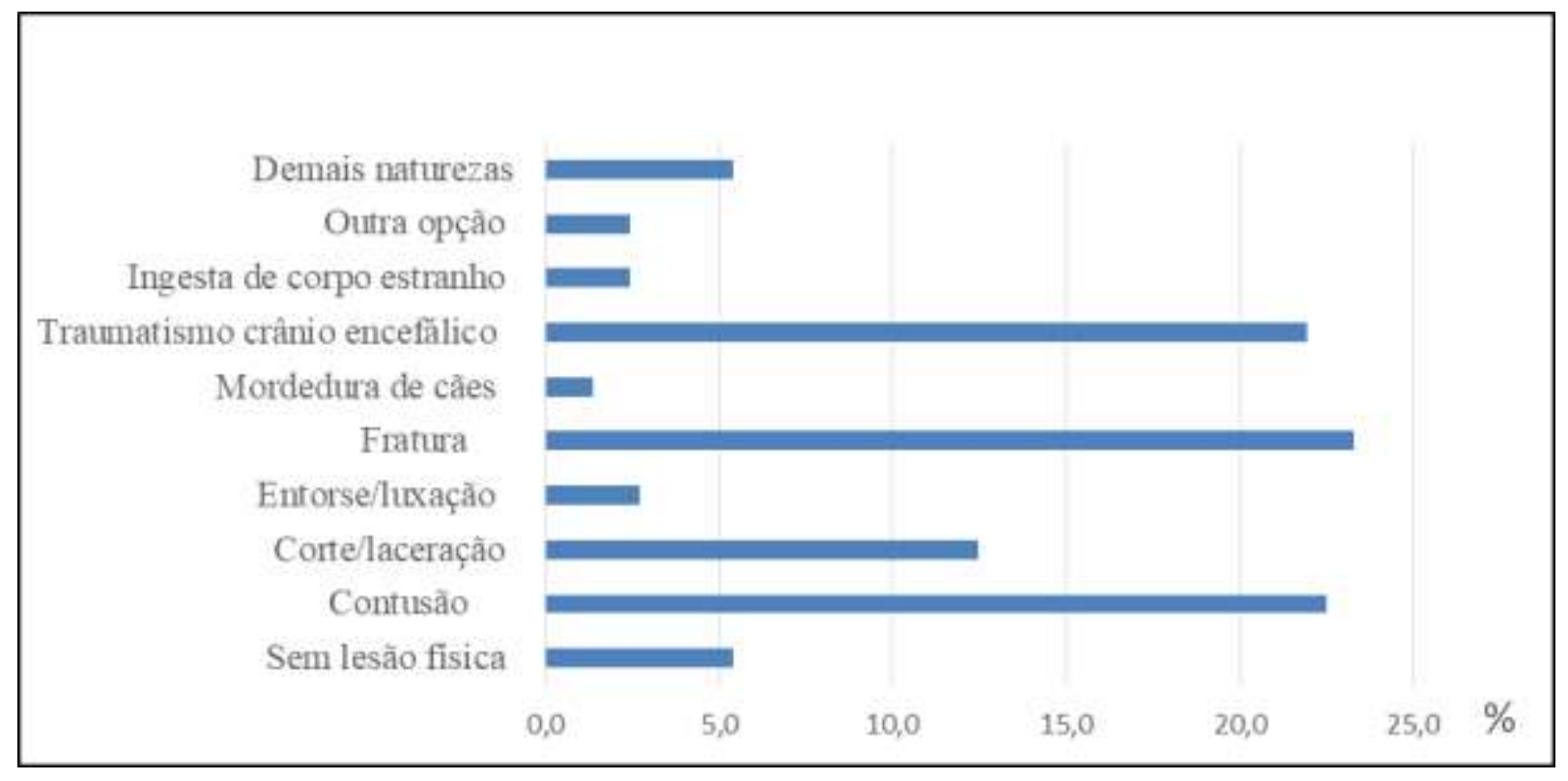

Fonte: Dados da pesquisa (2020).

Das entrevistas, quando perguntado aos pais ou responsáveis se achavam que o acidente poderia ter sido prevenido, 43,5\% responderam que não. Quando questionado se já ouviram falar sobre prevenção de acidentes, 52,2 \% disseram que sim.

Por meio das entrevistas realizadas, foi possível identificar que alguns pais recebem orientações preventivas quando as crianças eram bebês, e à medida que elas crescem essas orientações deixam de ser frequentes. Outra ressalva é que embora recebam informação, de como prevenir incidentes, acabam não as executando, acreditando não achar necessário ou que o evento está longe de sua realidade.

Das entrevistas aplicadas, foi observado que as crianças, em sua maioria, estavam sob supervisão de adultos, porém como as mesmas estavam brincando os pais ou acompanhantes julgaram que o acidente não poderia ter sido evitado.

Conforme elucidado na Figura 4,71,5\% das crianças evoluíram para alta hospitalar, 13,8\% permaneceram em observação seguida de alta e em 4,9\% dos casos foi necessária alguma intervenção cirúrgica. Este desfecho foi avaliado dentro das 24 horas após o acidente.

A partir dos resultados evidenciados e levantamento do perfil de acidentes mais prevalentes em crianças de zero a seis anos completos na referida instituição, foi elaborado um material educativo, a ser utilizado pelos profissionais de saúde, tanto a nível hospitalar como na Atenção Primária em Saúde, para orientação aos pais, familiares e cuidadores de crianças na prevenção de futuros acidentes. Conforme figura 2. 
Research, Society and Development, v. 10, n. 2, e21010212415, 2021

(CC BY 4.0) | ISSN 2525-3409 | DOI: http://dx.doi.org/10.33448/rsd-v10i2.12415

Figura 2. Material educativo para prevenção de acidentes na infância, Curitiba, 2020.
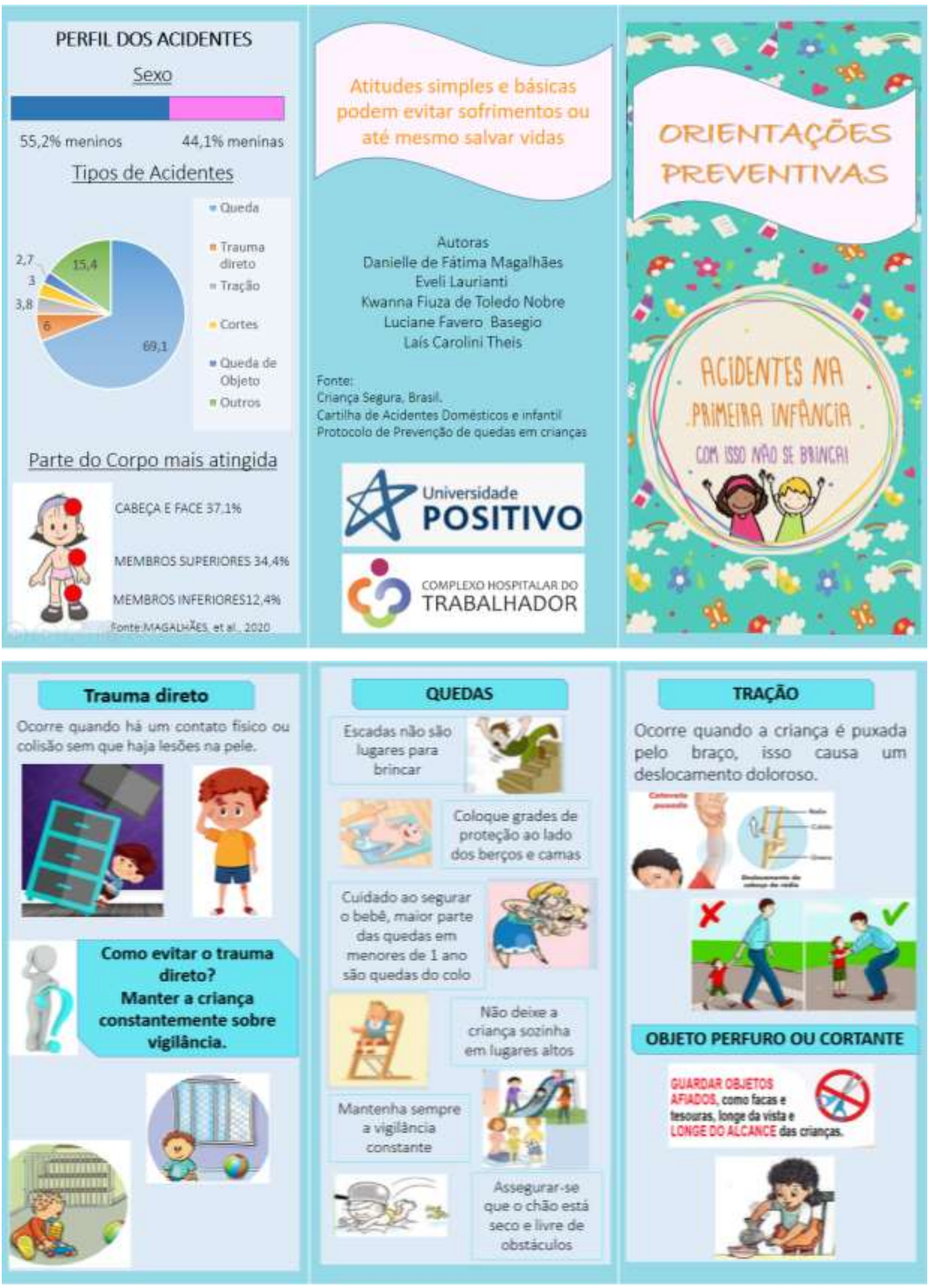

Fonte: Autoras (2020). 


\section{Discussão}

Por meio dos dados levantados foi possível identificar o perfil das crianças e dos acidentes ao qual foram submetidas. Destaca-se maior prevalência do sexo masculino, justificável, devido ao aspecto comportamental entre meninos e meninas. Os meninos, de forma geral, são mais ativos, buscam mais aventuras, se envolvem em atividades com velocidade, possuem uma maior liberdade, agressividade e brincadeiras com contato físico. Em contrapartida, as meninas possuem comportamento mais brando e brincadeiras amenas, além de estarem submetidas a uma maior vigilância por parte dos adultos decorrente de uma questão cultural, social e educacional (Brito, 2017; Filócomo et al., 2017; Silva et al., 2017a; Silva et al., 2017b; Simas \& Souza, 2019).

Observou-se que crianças com faixa etária de três anos foram as mais acometidas, isso ocorre por estarem em processo de desenvolvimento da coordenação motora e por incapacidade em reconhecer os perigos que as cercam (Criança Segura, 2020). Esta afirmativa vai ao encontro com um estudo descritivo de Araújo et al (2017), em que houve uma análise da interpretação dos sentidos com crianças em fase pré-escolar, que comprovam, que por estarem em desenvolvimento da cognição, interação social e do sistema motor, não sabem distinguir adequadamente riscos.

A residência foi elencada como principal local dos acidentes. Considerando que mais de $30 \%$ dos acidentes acontecem em casa e que, no momento atual de enfrentamento de uma pandemia, em que as crianças estão em tempo integral em suas moradias, é compreensível que esse seja o local onde as crianças sejam mais acometidas. Por esta mesma razão, houve também, uma semelhança entre os dias da semana, uma vez que as crianças permanecem em casa diariamente (Criança Segura, 2020).

Evidenciou-se que a queda foi o principal tipo de injúria não intencional, com elevada incidência para esse grupo etário. Tal achado condiz com outros estudos que trazem a queda como predominância de acidente nessa faixa etária (Barcelos et al., 2017; Faria et al., 2018; Filócomo et al., 2017; Santos, 2018;). Isso se dá devido ao fato, de que as crianças estão em processo de desenvolvimento e crescimento. Já as crianças menores possuem outra característica, que é a cabeça desproporcional ao tamanho do seu corpo, o que favorece o desequilíbrio (Criança Segura, 2020).

No que diz respeito aos tipos de queda, este estudo diverge com a literatura, pois este traz a queda de outros níveis, como de maior predominância, seguida de queda da própria altura. Uma fração das quedas evidenciadas está relacionada aos tipos de brincadeiras realizadas pelas crianças, como, por exemplo, as que utilizam motinho/triciclo, escorregador, balança e cama elástica (Amorim, 2017; Brito, 2017).

Fratura, contusão e trauma cranioencefálico foram à natureza da lesão mais predominante, atingindo cabeça e face, membros superiores e inferiores, resultado este coerente com demais estudos com o mesmo objeto de pesquisa (Amorim, 2017; Brito, 2017; Simas \& Souza, 2019). Vale ressaltar que crianças menores de dois anos possuem desproporção das dimensões de cabeça e corpo, facilitando lesões nesse segmento corpóreo (Filócomo et al., 2017).

Corroborando com a literatura, o desfecho do atendimento nas primeiras 24 horas acarretou em alta hospitalar, sem necessidade de intervenção ou internação, seguido de uma parcela de observação e cirurgia (Amorim, 2017; Brito, 2017; Filócomo et al., 2017; Santos, 2018).

A educação em saúde estimula a prevenção de acidentes e a promoção da saúde para um desenvolvimento infantil saudável visando, evitar possíveis sequelas que podem comprometer a criança que está em pleno desenvolvimento. Práticas preventivas e fiscalização devem ser atribuídas com a mesma prioridade dos demais assuntos que envolvem a qualidade de vida e o desenvolvimento infantil saudável (Filócomo et. al., 2017; Silva et. al., 2017b).

Destaca-se ainda a importância dos profissionais de saúde que assistem as crianças em unidades de pronto atendimento, estejam capacitados tanto para as ações de urgência e emergência, bem como para exercer esse papel de educador em saúde. Considerando o quanto é essencial que o profissional que trabalha nessa área estar especificadamente 
capacitado e com uma vasta experiência de atuação com pacientes críticos, os serviços de atenção em urgência e emergência devem estimular o uso de todo conhecimento visando utilizar os recursos disponíveis, tendo em vista que os mesmos são limitados, principalmente no serviço de atendimento pré-hospitalar que não possui a sua disposição os mesmos recursos que uma unidade hospitalar, além de que, a quantidade de profissionais atuantes em determinado socorro é restrita, dessa forma a Educação Permanente serve como estratégia para a otimização desse trabalho, pois esta promove melhorias na qualidadedo desempenho técnico do profissional, diminuindo o índice de falhas nos procedimentos

\section{Considerações Finais}

Esse estudo permitiu identificar o perfil e os principais acidentes que acometem as crianças na primeira infância, atendidas em uma instituição referência para o atendimento de traumas, o que possibilitou elaborar um material educativo, com orientações e medidas preventivas voltadas aos pais ou responsáveis, de forma a conscientizá-los e capacitá-los, com o intuito de evitar ou minimizar a incidência desses acidentes e os eventuais danos que podem afetar a saúde das crianças.

Entre as limitações para a realização do estudo, destaca-se principalmente a restrição de entrada no campo, uma vez que no tempo previsto para a coleta de dados, o mundo enfrentava (e ainda enfrenta) uma pandemia, o que acarretou no encurtamento do tempo de coleta de dados, tanto em prontuário quanto de entrevistas. Os prontuários eletrônicos e registros apresentavam ausência e/ou falhas de registros, fato que limitou o preenchimento do instrumento de forma integral.

O estudo contribui como um alerta para pais, responsáveis ou cuidadores de crianças e também para os profissionais de saúde, ao constatar que as crianças estão expostas diariamente a inúmeros riscos que podem levar a quadros leves, moderados e graves. Embora existam inúmeros fatores ambientais que favorecem os riscos, há uma falsa sensação de segurança quando se fala de acidentes em domicílio, ou em momentos de lazer.

Faz-se necessário que profissionais de saúde sejam mais proativos e promotores de informações, orientando os pais ou responsáveis sobre a prevenção de acidentes, discutindo este problema, no mesmo nível de prioridade que os demais assuntos que envolvam o desenvolvimento saudável da criança. Sendo imprescindível falar sobre prevenção de acidentes nas consultas médicas e de enfermagem durante a realização da puericultura; em ações preventivas e educativas nas escolas, e, até mesmo, desenvolver campanhas de conscientização e orientação sobre o assunto, a fim de disseminar conhecimento e reduzir agravos.

Deste modo, deseja-se que o material educativo elaborado possa contribuir para a disseminação de conhecimento e orientação aos pais e/ou responsáveis de crianças e que esses possam ser multiplicadores de informações para a comunidade, colaborando com as ações dos profissionais de saúde em ações educativas e preventivas. E recomenda-se estudos futuros que busquem conhecer a percepção dos pais e responsáveis acerca dos acidentes domésticos com intuito de conhecer suas percepções e entendimentos sobre a temática.

\section{Referências}

Amorim, E. S., Silva, A. R., Lima, E. O., \& Mendonça, P. M. R. (2017). Perfil epidemiológico de crianças vítimas de trauma cranioencefálico. Rev. Enfermagem UFPE online, 11(10), 4150-617.

Araújo, A. R., Gubert, F. A., Tomé M. A. B. G., Martins, M. C., Fontenele, N. L., \& Barros, E. C. (2017). Prevenção de acidentes em uma creche: experiência com pais, professores e pré-escolares. Rev. de Enfermagem UFPE online, 11(4), 1671-8.

Barcelos, R. S., Santos, I. S., Matijasevich, A., Barros, A. J. D., Barros, F. C., França, G. V. A., \& Silva, V. L. S. (2017). Acidentes por quedas, cortes e queimaduras em crianças de 0-4 anos: coorte de nascimentos de Pelotas, Rio Grande do Sul, Brasil, 2004. Cadernos de Saúde Pública, 33 (2), e00139115. 10.1590/0102-311x00139115.

Brito, M. A., Melo, A. M. N., Veras, I. C., Oliveira, C. M. S., Bezerra, M. A. R., \& Rocha, S.S. (2017). Fatores de risco no ambiente no ambiente doméstico para quedas em crianças menores de cinco anos. Rev. Gaúcha de Enfermagem, 38(3), e2017-0001. 10.1590/1983-1447.2017.03.2017-0001. 
Faria, C. G., Queiroz, D. B., Matias, O. M., \& Melo, T. P. (2018). Principais causas de internação por acidentes domésticos na infância em um hospital Universitário do Oeste do Paraná. Brazilian Journal of Surgery and Clinical Research, 22(2), 103-109.

Filócomo, F. R. F., Harada, M. J. C. S., Mantovani, R., \& Ohara, C. V. S. (2017). Perfil dos acidentes na infância e adolescência atendidos em um hospital público. Acta Paulista de Enfermagem, 30(3), 287-294. 10.1590/1982-0194201700044.

Ministério da Saúde. .(2017a). Acidentes e violência. Brasília, DF. http://www.saude.gov.br/saude-de-a-z/acidentes-e-violencias.

Ministério da Saúde. Conselho Nacional de Saúde. (2012). Resolução $n^{o}$ 466, de 12 de dezembro de 2012. Diretrizes e normas regulamentadoras de pesquisas envolvendo seres humanos. Diário Oficial da União, Brasília, DF.

Ministério da Saúde. Datasus. (2017b). Informações de saúde. Morbidade hospitalar de SUS por causas externas - por local de determinação. Brasília, DF. http://tabnet.datasus.gov.br/cgi/tabcgi.exe?sih/cnv/fiuf.def.

Ministério da Saúde. Datasus. (2017c). Informações de saúde. Óbitos por causas externas. Brasília, DF. http://tabnet.datasus.gov.br/cgi/tabcgi.exe?sim/cnv/ext10uf.def.

Ministério da Saúde. Secretaria de Atenção à Saúde. Departamento de Ações Programáticas Estratégicas. (2018). Política Nacional de Atenção Integral à Saúde da Criança: orientações para implementação. Brasília, DF.

Ministério da Saúde. (2019). Viva Inquérito 2017: Vigilância de Violências e Acidentes em Serviços Sentinelas de Urgência e Emergência - Capitais e Município.Brasília, DF. https://www.saude.gov.br/images/pdf/2019/dezembro/05/viva-inquerito-2017.pdf.

Pereira A. S., Shitsuka, D. M., Parreira, F. J., \& Shitsuka, R. (2018). Metodologia da pesquisa científica. [e-book]. Santa Maria. Ed. UAB/NTE/UFSM. https://repositorio.ufsm.br/bitstream/handle/1/15824/Lic_Computacao_Metodologia-Pesquisa-Cientifica.pdf?sequence=1 .

Rodrigues, G. V. B., Cortez, E. A., Almeida, Y. S., \& Santos, E. C. G. (2020). . Permanent health education in urgency and emergency services: an integrating review. Research, Society and Development, 9(8), p. e14985269. 10.33448/rsd-v9i8.5269.

Santos, T. C., Ghisi, G. C., Fachini, J. S., Dias Junior, G., \& Santos Junior, J. R. (2018). Perfil epidemiológico das internações por acidentes domiciliares em um hospital pediátrico da região Sul do Brasil. Arquivos Catarinenses de Medicina, 47(4), 29-38.

Silva, L. S. R., Silva, T. A., Santos, C. M., Pereira, L. R. S., Correia, N. S., \& Silva, A. C. A. (2017a). Mortalidade infantil relacionada a diversos tipos de acidentes por causas externas. Rev. de Enfermagem UFPE online, 11(5), 2098-105.

Silva, M. F., Fontinele, D. R. S., Oliveira, A. V. S., Bezerra, M. A. R., \& Rocha, S. S. (2017). Fatores determinantes para a ocorrência de acidentes domésticos na primeira infância. Journal of Human Growth and Development, 27(1), 10-18. 10.7322/jhgd.127643.

Silva, J. S., \& Fernandes, K. S. (2019). Acidentes domésticos mais frequentes em crianças. Trabalho de Conclusão de Curso (Bacharelado em Enfermagem), Centro Universitário do Planalto Central Aparecido dos Santos, Brasília, 2019.

Simas, V. F. C., \& Souza, A. S. (2019). Crianças hospitalizadas vítimas de acidentes na primeira infância. Revista Pró-UniverSUS, 10, (1), 25-28.

World Health Organization (WHO). (2009). Glossary of Patient Safety Concepts and References. The Conceptual Framework for the International Classification for Patient Safety, Technical Annex 2, version 1.1. https://www.who.int/patientsafety/taxonomy/icps_technical_annex2.pdf. 